\title{
Fetal Alcohol Syndrome
}

\author{
G Jayanthi Babu ${ }^{1}$, Annie Annal M², Umamaheswari Ramesh ${ }^{3}$
}

\begin{abstract}
Alcohol consumption during pregnancy can have adverse health consequences for the developing fetus and therefore is a significant public health problem. During pregnancy, there is no proven safe level of alcohol consumption. The term fetal alcohol syndrome (FAS) is a medical diagnosis focused on a unique combination of physiological, psychological, and intellectual disorders triggered by prenatal exposure to alcohol. Fetal alcohol syndrome is an unavoidable, fatal condition which impacts each dimension of a child's life. Thus, it is a very fundamental requisite for the caregivers and healthcare personnel to recognize the symptoms of FAS earlier and ensure the optimal growth of the child.

Keywords: Developmental delay, Fetal alcohol syndrome, Pregnancy.

Pondicherry Journal of Nursing (2019): 10.5005/jp-journals-10084-12132
\end{abstract}

\section{INTRODUCTION}

Since the beginning of recorded history, alcoholic drinks and the issues they create have been common fixtures in human societies. Problems related to alcohol were primarily male-focused. ${ }^{1}$ However, recent research has shown that while less women consume alcohol than men, with the same amount of alcohol consumed, the health and other effects of women's alcohol intake could be greater than that of men. ${ }^{2}$ This may damage the fetus, giving rise to a series of genetic abnormalities called fetal alcohol syndrome (FAS). ${ }^{3}$ Fetal alcohol syndrome is one of a spectrum of disorders under fetal alcohol spectrum disorders (FASDs). ${ }^{4}$

\section{Definition}

Fetal alcohol syndrome is highly variable group of birth defects including intellectual disability, deficient growth, and malformations of the skull and face that tend to occur in the offspring of women who consume large amounts of alcohol during pregnancy. ${ }^{5}$

\section{INCIDENCE}

It is estimated that at least one million people in this country have FAS and approximately five million have partial FAS and (other) fetal alcohol spectrum disorders. ${ }^{6}$

\section{Causes}

All conditions involving fetal alcohol spectrum disorders derive from one common cause, which is prenatal alcohol exposure. Alcohol is extremely teratogenic during all three trimesters to a fetus.

- Women over 30 years with a long history of alcohol are more likely to give birth to a child with FAS.

- Having one child with FAS raises the risk for children subsequently.

- Women with genetic susceptibility may be at a higher risk. ${ }^{7}$

\section{Signs And Symptoms}

It also includes birth defects like abnormalities of the kidneys, eyes, hearing loss, and heart defects (Fig. 1). ${ }^{8}$
${ }^{1-3}$ Department of Obstetrics and Gynecology Nursing, Kasturba Gandhi Nursing College, Sri Balaji Vidyapeeth, Puducherry, India

Corresponding Author: Umamaheswari Ramesh, Department of Obstetrics and Gynecology Nursing, Kasturba Gandhi Nursing College, Sri Balaji Vidyapeeth, Puducherry, India, Phone: +91 9965228299, e-mail: umamaheswarir@kgnc.ac.in

How to cite this article: Babu GJ, Annie Annal M, Umamaheswari R. Fetal Alcohol Syndrome. Pon J Nurs 2019;12(4):93-95.

Source of support: Nil

Conflict of interest: None
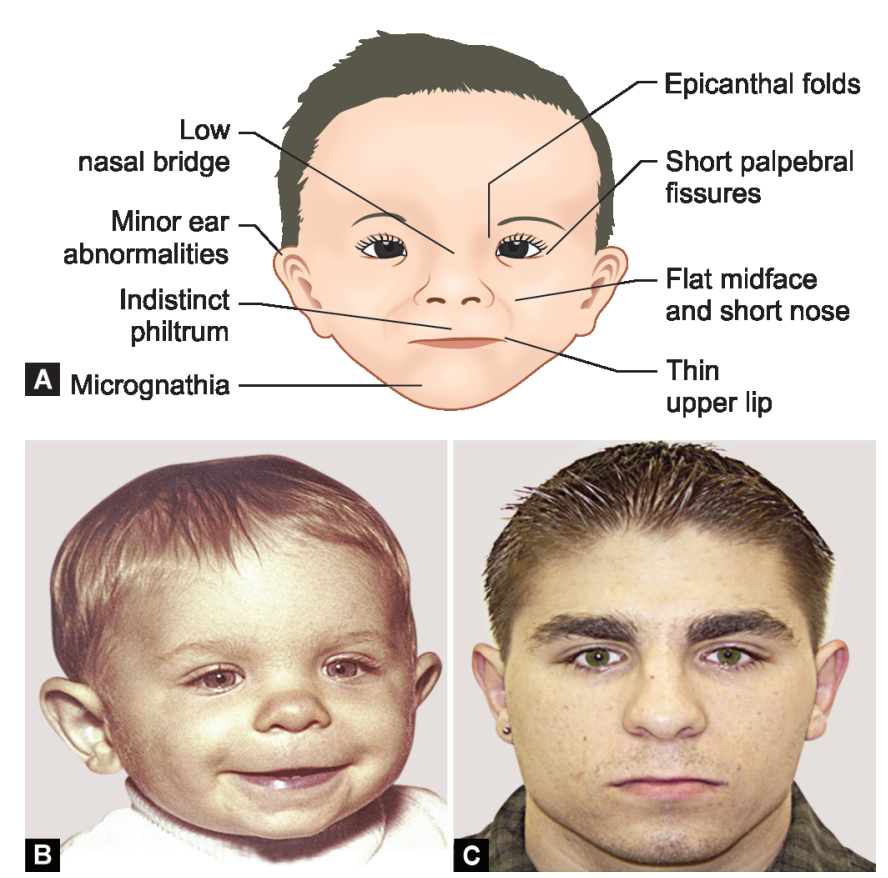

Figs $1 \mathrm{~A}$ to $\mathrm{C}$ : Facial signs of fetal alcohol syndrome

() The Author(s). 2019 Open Access This article is distributed under the terms of the Creative Commons Attribution 4.0 International License (https://creativecommons. org/licenses/by-nc/4.0/), which permits unrestricted use, distribution, and non-commercial reproduction in any medium, provided you give appropriate credit to the original author(s) and the source, provide a link to the Creative Commons license, and indicate if changes were made. The Creative Commons Public Domain Dedication waiver (http://creativecommons.org/publicdomain/zero/1.0/) applies to the data made available in this article, unless otherwise stated. 


\section{Diagnosis}

\section{History Taking}

Prenatal exposure to alcohol is measured by questioning the biological mom or other family members related to

- Drug use during gestation, maternal clinical reports (if available), and examination of appropriate birth records, court records (if applicable), records of substance dependency interventions, substance biomarkers, or other credible sources.

- If the exposure level is confirmed, then the amount, frequency, and timing of prenatal alcohol use can dramatically impact the other features of FASD. ${ }^{9}$

\section{Assessment}

The diagnosis of FAS by doctors looks for findings based on the following three areas:

- Characteristic facial anomalies (physical examination),

- Growth retardation (mental status examination),

- Central nervous system (CNS) involvement (neurological examination). ${ }^{10}$

There is no laboratory test which could confirm FAS. A lot of its symptoms look like attention deficit hyperactivity disorder (ADHD). ${ }^{11}$

\section{Complications}

Many of the difficulties that infants and children with FAS face persist into their adolescence and adulthood. These may include

- "Regulation" issues (sleep, treatment, and excitement),

- Cognitive disabilities,

- Sight and auditory loss,

- Intellectual retardedness,

- Memory, thought and decision deficits, and

- Short stature. ${ }^{8}$

\section{TREATEMENT}

CNS damage from exposure to prenatal alcohol is severe, and there is no treatment for fetal spectrum disorders. Symptoms of FAS cannot be healed, but early diagnosis and treatment can improve a child's growth and outlook.

One of the most common approaches is to treat by home care to integrate developmental and educational services. Children can do better when they

- are diagnosed before age 6,

- are in a supportive, responsible, and healthy family during their school years,

- not immune to abuse,

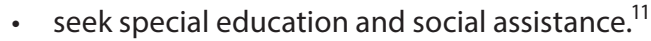

\section{FolLow-Up}

Children with FAS should undergo repeated examinations as follows:

- Patients were subjected to medical, neurological and clinical examinations, EEG records, and psychological assessments.

- Different subgroups of these children were re-examined after a period of 3-4 years.
- Follow-up examinations may reveal that with increasing age dysmorphic signs became less apparent in children with FAS.

- Neurologic performance may improve.

- And EEG recordings revealed less pathological patterns. ${ }^{12}$

\section{Individual Education Program}

Women are encouraged to consult with their obstetricians, pediatricians, and other healthcare providers when attempting to conceive (prenatally) in order not just to consider the complications but also to make the right decision for their baby's safety. ${ }^{13}$

Children with delayed skills or other disabilities may be eligible for special services, free of charge to families, that provide individualized education programs in public schools. Knowing how parents can navigate services like that can help them become better advocates for their kids. A child with learning and coping problems, and known as a student with special needs, is the ideal candidate for an individual education program (IEP).

The first step is to obtain detailed data about the progress of the student by evaluating the student and assessing the performance of the student (attention, actions, completion of the job, examinations, classwork, homework, etc.)

The next step is an IEP meeting where the staff and the parents discuss what is going to head into the program. The IEP cover page details the support services your child will receive and how frequently they will be provided (for example, twice a week for occupational therapy). Special education, speech therapy, occupational or physical therapy, rehabilitation, audiology, medical care, nursing, and vision or hearing therapy may be part of the support services. Individual education program can also be changed on an as-needed basis at any time. The IEP mechanism is complicated, but it is also an important way to improve the learning and functioning of children. ${ }^{14}$

\section{Prevention}

Prevention of FAS is the responsibility of all healthcare workers to enhance healthcare team outcomes. The diagnostic team includes

- A pediatrician and/or physician who may have experience in fetal alcohol spectrum disorders,

- Nurse practitioner,

- Social worker,

- Occupational therapist,

- Speech-language pathologist,

- Psychologist, and

- Parent.

In certain cases, exposure to prenatal alcohol is accidental, because women continue their usual habit of drinking until they know they are pregnant. Clinicians should be fully aware that FAS is preventable and it should be advised to the couples in prenatal diagnosis of pregnancy. ${ }^{4}$

\section{Conclusion}

Consuming alcohol during pregnancy can cause a group of conditions called FASDs. Children who are born with FASD can have a mix of problems, such as medical, behavioral, educational, and social problems. ${ }^{15}$ The commonly accepted estimate for the USA of 10 per 1000 children affected was derived from clinic-based studies or studies of single communities using small samples. ${ }^{7}$ However, FAS is $100 \%$ preventable if a woman does not drink alcohol while 
she is pregnant. ${ }^{16}$ So, primary care providers should consider the possibility for FASD whenever a child has suggestive physical stigmata and/or is being assessed for poor growth, developmental delays, or behavioral concerns, including attention deficit or school failure. $^{17}$

\section{References}

1. Wilsnack RW, Wilsnack SC, Obot IS. Why study gender, alcohol and culture? In: Obot IS, Room R. Alcohol, gender and drinking problems (GENACIS): Perspectives from low and middle income countries. Geneva: World Health Organization; 2005. pp. 1-23.

2. Murthy NV, Benegal V, Murthy P. Alcohol dependent females: a clinical profile. Available at http://www.nimhans.kar.nic.in/deaddiction/lit/ Female\%20Alcoholics.pdf. Accessed on May 7, 2008.

3. Home. https://indianpediatrics.net/dec2008/dec-977-983.htm.

4. Demetrios V, Bernstein B. Fetal alcohol syndrome. StatPearls. StatPearls Publishing; 2020.

5. Definition of Fetal Alcohol Syndrome. https://www.merriam-webster. com/dictionary/fetal+alcohol+syndrome.

6. "WHO | Fetal Alcohol Syndrome: Dashed Hopes, Damaged Lives." WHO, https://www.who.int/bulletin/volumes/89/6/11-020611/en/.

7. May PA, Chambers CD, Kalberg WO, et al. Prevalence of fetal alcohol spectrum disorders in 4 US communities. JAMA 2018;319(5):474-482. DOI: 10.1001/jama.2017.21896.
8. "Fetal Alcohol Syndrome (FAS) Treatment, Symptoms \& Causes." MedicineNet, https://www.medicinenet.com/fetal_alcohol_ syndrome_fas/article.htm. Accessed 28 Feb. 2020.

9. "Fetal Alcohol Spectrum Disorder." Wikipedia, 24 Feb. 2020. Wikipedia, https://en.wikipedia.org/w/index.php?title=Fetal alcohol_spectrum_disorder\&oldid $=942438714$.

10. Fetal Alcohol Syndrome: Background, Pathophysiology, Epidemiology. Nov. 2019. eMedicine, https://emedicine.medscape. com/article/974016-overview.

11. "Fetal Alcohol Syndrome." WebMD, https://www.webmd.com/baby/ fetal-alcohol-syndrome.

12. Spohr HL, Steinhausen HC. Follow-up studies of children with fetal alcohol syndrome. Neuropediatrics 1987;18(1):13-17. DOI: 10.1055/s2008-1052428.

13. "Prevention-Education-and-Counseling." AAP.Org, http://www. aap.org/en-us/advocacy-and-policy/aap-health-initiatives/fetalalcohol-spectrum-disorders-toolkit/Pages/Prevention-Educationand-Counseling.aspx.

14. Individualized Education Programs (IEPs) (for Parents) - Nemours Kids Health. https://kidshealth.org/en/parents/iep.html?WT.ac=p-ra.

15. Fetal Alcohol Spectrum Disorders. https://medlineplus.gov/ fetalalcoholspectrumdisorders.htm.

16. "Definition of Fetal Alcohol Syndrome." MedicineNet, https://www. medicinenet.com/script/main/art.asp?articlekey=3415.

17. Williams JF, Smith VC. Fetal alcohol spectrum disorders. Pediatrics 2015;136(5):E1395-E1406. DOI: 10.1542/peds.2015-3113. 